calprotectin testing and secondary care straight-to-test may impact diagnostic delays.

\section{PTU-109 DEVELOPMENT AND IMPLEMENTATION OF THE UK NATIONAL ENDOSCOPY DATABASE (NED)}

1,2Keith Siau*, ${ }^{3}$ Thomas JW Lee, ${ }^{4}$ Shiran Esmaily, ${ }^{5}$ James Docherty, ${ }^{6} J o h n$ Stebbing, ${ }^{7}$ Matthew J Brookes, ${ }^{1}$ Raphael Broughton, ${ }^{8}$ Peter Rogers, ${ }^{9}$ Paul Dunckley, ${ }^{4,10,11}$ Matthew D Rutter. 'Joint Advisory Group, London, UK; ${ }^{2}$ University of Birmingham, Birmingham; ${ }^{3}$ North Tyneside General Hospital, North Shields; ${ }^{4}$ University Hospital of North Tees, Stockton-on-Tees; ${ }^{5}$ Raigmore Hospital, Inverness; ${ }^{6}$ Royal Surrey County Hospital, Guildford; ${ }^{7}$ New Cross Hospital, Wolverhampton; ${ }^{8}$ Weblogik Ltd, Ipswich; ${ }^{9}$ Gloucestershire Royal Hospitals NHSFT, Gloucester; ${ }^{10}$ Durham University, Stockton-on-Tees; ${ }^{11}$ Newcastle University, Newcastle-Upon-Tyne

\subsection{6/gutjnl-2019-BSGAbstracts.468}

Introduction The UK National Endoscopy Database (NED) is a JAG-led informatics project centred on the extraction of data from endoscopy reporting systems (ERS) in participating units to a centralised database. The aim of NED is to facilitate quality assurance, service intelligence, research and training in endoscopy. Herein, we present an overview of the development and implementation of NED from its inception to date, with updates on future directions.

Methods The NED project began in 2013 with the support of UK stakeholder societies. ERS companies were collectively approached to engage in software modification to enable automated uploads of a standardised minimum dataset to NED without patient identifiable data. Since the national roll-out of pilot NED in 2017, endoscopists and organisational leads from services uploading to NED have been able to review key performance indicators on a web-based platform for quality assurance.

Results As of February 2019, 339 out of a total of 529 JAGregistered UK endoscopy units (64\%) have actively uploaded to NED. The database comprises over 800,000 procedures (gastroscopy: 348,000; sigmoidoscopy: 282,000; colonoscopy 167,000; ERCP: 12,000) and presents procedure-specific KPIs which can be accessed at individual, unit, regional and national levels. Trends in procedural uploads since 2017 are shown in Abstract PTU109 Figure 1. The functionality of mapping trainee and trainer specific data into the JETS training e-portfolio is undergoing final phase validation, with the objective of providing reliable and unbiased training data to support training and certification. Consultation with multisociety stakeholders and ERS companies on additional data points for NED iteration 2 (NEDi2) is underway. These are intended to expand on existing KPIs, in addition to providing support for polypectomy, ERCP and EUS in line with UK-relevant quality standards.

Conclusions With the engagement of ERS companies, it is possible to implement an automated system to extract data from individual endoscopy units to a centralised database. This has the potential to facilitate endoscopy quality assurance and research at a national level.

\section{Education \& Training}

\section{Orals}

\section{OWE-34 ADR IN CRC SCREENING PROGRAMS: MASTER SHOW ME THE WAY TO BECOME A HIGH DETECTOR!}

${ }^{1}$ Erik Rosa-Rizzotto*, ${ }^{1}$ Diego Caroli, ${ }^{2}$ Fabio Monica, ${ }^{3}$ Saia Mario, ${ }^{3}$ Manuel Zorzi, ${ }^{3}$ Giulia Capodaglio, ${ }^{2}$ Anna Turrin, ${ }^{3}$ Elena Narne, ${ }^{1}$ Franca De Lazzari, Endoscopists Group Veneto Colorectal Cancer Screening2. ' 5 St. Anthony Hospital, Padova, Italy; ${ }^{2}$ Gastroenterology Unit, Cattinara Hospital, Trieste, Italy; ${ }^{3}$ Azienda Zero, Padova, Italy

\subsection{6/gutjnl-2019-BSGAbstracts.469}

Background Although the adenoma detection rate (ADR) cutoff for colonoscopies after positive fecal immunochemical tests (FIT) continues to be a debated issue, the ADR is one of the most accepted benchmarks for colonoscopy quality. There is some evidence that the quality of colonscopy screening can be improved by educational interventions. This study aimed to analyze the effect of endoscopist-related characteristics on colonoscopy quality indicators and to evaluate the factors affecting ADR patterns registered in the colorectal (CRC) screening program in the Veneto Region between 201-017.

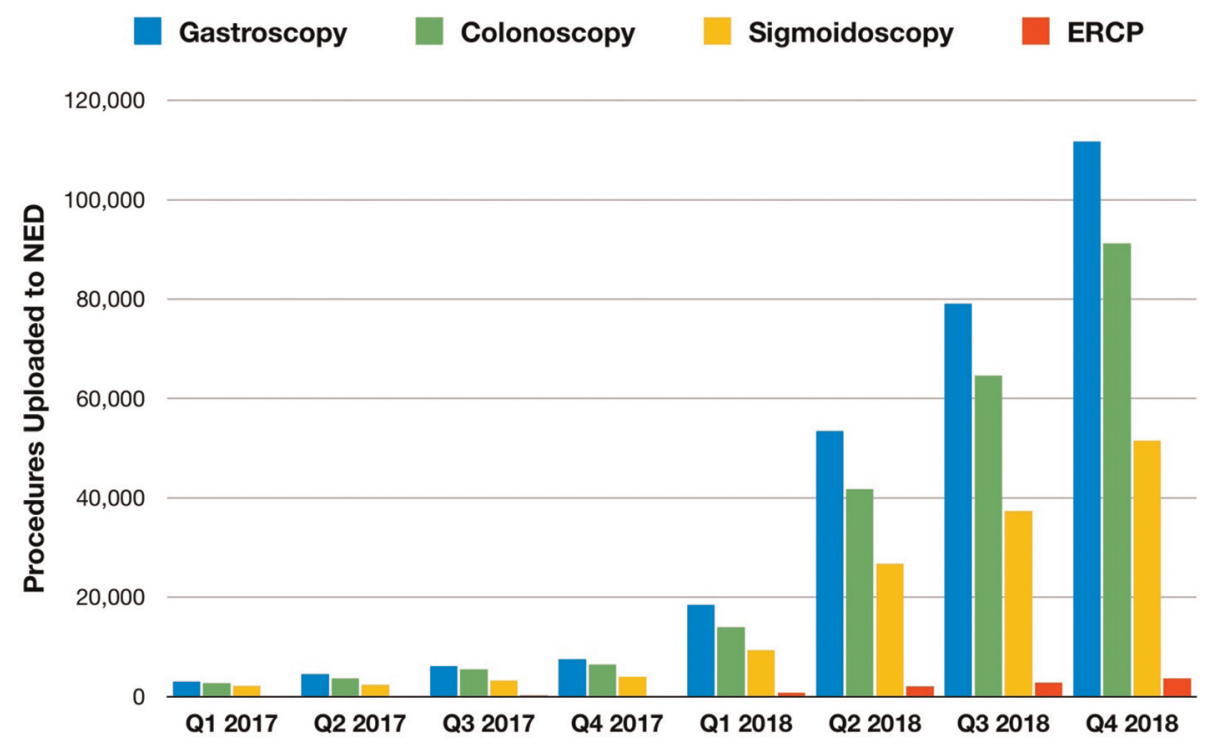

